\title{
Footprints of dioxins in global pork meat trade
}

\author{
Authors: Jianmin $\mathrm{Ma}^{1}$, Kaijie Chen ${ }^{1,}$ Tao Huang ${ }^{2}$, Shu Tao ${ }^{1}$ \\ ${ }^{1}$ College of Urban and Environmental Sciences, Laboratory for Earth Surface Processes, Peking University, \\ Beijing 100871, China \\ ${ }^{2}$ Key Laboratory for Environmental Pollution Prediction and Control, Gansu Province, College of Earth and \\ Environmental Sciences, Lanzhou University, Lanzhou 730000, PR China \\ Polychlorinated dibenzo-p-dioxins/furans (PCDD/Fs), known as dioxins, are one of the most toxic chemicals. Their \\ persistence in the environment and lipophilic property result it their bioaccumulate and biomagnify in food webs, which \\ pose potential health risks to humans. Establishment of dioxin inventory is a crucial step toward elimination of worldwide \\ dioxins contaminations and risk assessment. In this study, based on available national dioxin inventories worldwide, and \\ human social economic activities factors related to PCDD/Fs emission using principle component analysis (PCA), we \\ established a multiple linear regression model to estimate the global atmospheric PCDD/Fs emissions by country. Then, \\ gridded population density was used as surrogate data to construct a global gridded emission inventory for PCDD/Fs with $1^{\circ}$ \\ longitude by $1^{\circ}$ latitude resolution. Using this emission inventory CanMETOP (Canadian Model for Environmental \\ Transport of Organochlorine Pesticides) model was employed to conduct multiple scenario simulations of the atmospheric \\ transport between the Northern and Southern Hemisphere, and to quantify the effect of pork trade on human exposure to \\ dioxins. The results show that global atmospheric PCDD/Fs emission decreased from 2002 to 2012. Higher emissions of \\ $\mathrm{PCDD} /$ Fs were identified in Asia, Africa, and Europe. Of which China is the largest emitter, releasing approximately $4.5 \mathrm{~kg}$ \\ TEQ PCDD/Fs in 2012. It was found that PCDD/Fs were almost not exchanged between the Northern and Southern \\ Hemisphere through atmospheric transport. We assessed the human exposure to PCDD/Fs embodied in global pork meat by \\ combining CanMETOP modelled concentrations in air, soil, water with a terrestrial food web models. We examined the \\ origins of pork production where high dioxin emissions lead to high dioxin levels in the pork meats, and human exposure \\ to dioxins in the destination countries by pork consumptions imported from high dioxin emission countries. The \\ considerable higher exposure were found in the pork meat trade between the Southern and Northern Hemisphere as \\ compared with atmospheric transport and depositions, suggesting that the food trade is a major pathway of PCDD/Fs \\ transport between pork export and import countries, and between the Northern and Southern Hemisphere, rather than \\ atmospheric transport.
}

